\title{
Review of: "Empirical estimates of the mutation rate for an alphabaculovirus"
}

\author{
Xiulian Sun $^{1}$ \\ 1 Wuhan Institute of Virology
}

Potential competing interests: The author(s) declared that no potential competing interests exist.

This article is small and precise, focuses to answer one question, that is, the mutation rate of AcMNPV. The experimental design skillfully regarded the bacterial sequence in AcBAC as a neutral selection region (similar to a housekeeper gene), and constructed a recombinant virus with POLH. Through continuous passaging for 5 generations, SNV mutation was analyzed to estimate the mutation rate of the whole AcMNPV genome, so as to provide a theoretical basis for calculating the evolution time of other baculoviruses. The highlight lies in its algorithm calculation, and the calculated mutation rate is similar to that of other DNA/RNA (representative strain enterobacteria phage T2, Escherichia virus $\lambda$ ), thus the mutation rate is persuasive.

There are concerns with this study which include the following:

1. The effect of the 11.6 KB bacterial sequence region inserted into AcBAC on the mutation rate of the whole virus genome is unknown, it is only a neutral mutation region in theory. It is more convincing if the wild-type AcMNPV passing through 5 generations alone is set as control, and there was no significant difference between the mutation rate of AcBAC and wild-type AcMNPV passing through 5 generations.

2. In the background part, there is little introduction to the background related to mutation. For those without relevant professional knowledge, it is a little abstruse and difficult to understand. 